\title{
Fotografie riprese ad alta quota per mezzo di palloni durante l'eclisse totale di sole del 15-2-1961
}

\author{
R. Cialdea - M. Cimixo - G. Fei
}

Ricovuto il 15 Giugno 1961

In occasione dell'eclisse totale di sole del $15 \mathrm{Febblatio} \mathrm{1961,} \mathrm{l'Istituto}$ Nazionale di Geofisica, l'Osservatorio Astronomico di Roma su M. Mario ed il Ministero della Difesal Aeronantica hanno elaborato un piano di ricerche allo seopo di rilevare la luce diffusa da parte dell'atmosfera e di eseguire fotografie della corona estema del sole ad alta quota. La ricerea è stata effettuata inviando nell'alta atmosfera un apparato fotografico dello stesso tipo all-sky di quello già adoperato in occasione dell'eclisse totale di sole del $1952\left(^{1}\right)$.

L'appanato consiste in una macchina da ripresa, diretta verso il basso, che riprende l'intera volta celeste riflessa da uno specchio convesso $\left({ }^{2}\right)$. La macchina adoperata, del formato di $16 \mathrm{~mm}$, ha una velocità di ripresa di due fotogremmi al secondo con un tempo di esposizione di $1 / 8$ di secondo; un tempo di esposizione cosi elevato è necessario per poter eseguire valutazioni fotometriche della luce diffusa anche da parte dellaalta atmosfera durante la fase di totalità. Davanti allobiettivo una serie di quattro filtri ruota sincronicamente alla pellicola allo scopo di ottenere delle serie successive di fotogrammi con esposizioni diverse: in tal modo si può ottenere una più accurata misura fotometrica. Cn sistema ad orologeria assicura l'inizio della ripresa (dopo 45' di volo) e lo sgancio del pallone dopo un'ora e mezza dal lancio. Dal momento dello sgancio (che in occusione dell'eclisse doveva avvenire intorno ai 20-25 km di altezza) l'apparato scende in caduta libera fino a quota 3000 , dove un mecanismo barometrico fa aprire un paracadute per poter permettere il recupero di tutti gli apparati. Allo scopo di proteggere i delicati meccanismi di orologeria e di avanzamento del film dalle bassissime temperature dell'alta atmosfera, l'apparato è protetto da una opportuna scatola formata da materiale ad elevato isolamento termico. 
La quota è registrata su ogni fotogramma, fotografando un altimetro sistemato vicino allo specohio. Nella Fig. 1 è rappresentato tut to l'apparato: si individua a partire dall'alto il paracadute, la scatola isolante, e internamente ad essa il meccanismo ad orologeria, le batterie, la macchina da ripresa, ed esternamente, la scautola dei filtri, lo specchio e l'altimetro.

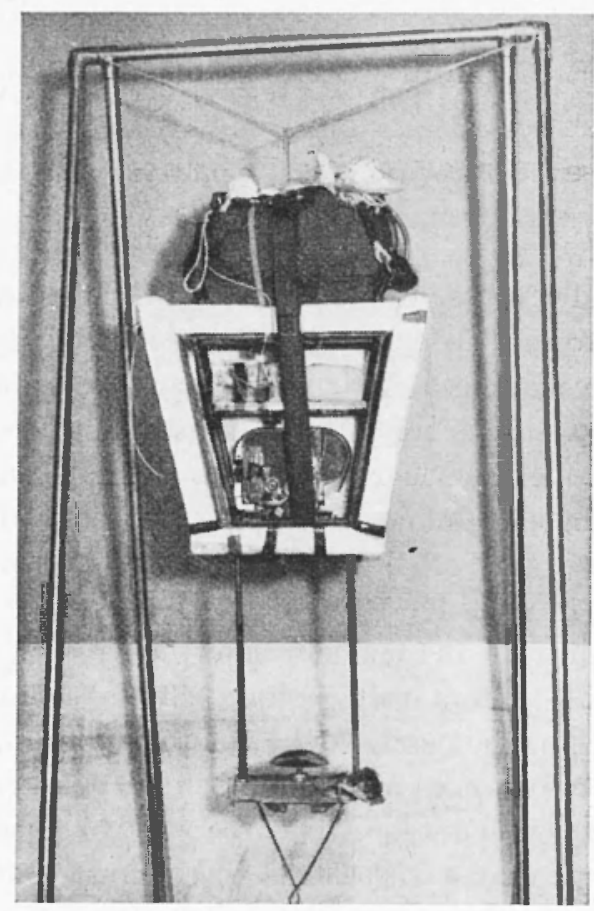

Fig. I

L'apparecchiatura, dato il suo notevole peso, è stata affidata a due palloni in parallelo: essa è partita il 15 mattina alle 7,47 (T.M.T.C.) dall'weroporto di Pisa. Il volo si è svolto regolarmente e si è raggiunta la quota massima di 24.000 metri; la discesa è avvenuta lentamente senza paracadute, in quanto a questa quota uno di due palloni è scoppiato mentre l'altro ha funzionato da freno nella discesa. La velocità di salita dell'apparato è stata però più bassa del previsto, cosicché al momento della totalità i palloni si trovavano alla quota di 9.500 metri. L'apparato ha funzionato regolarmente e la fase di totalità è stata integralmente ripresa. I tempi di esposizione, di necessità lunghi, sono risultati troppo 
grandi in relazione ai periodi medi degli irregolari moti oscillatori dell'apparato, moti dovuti alla turbolenza atmosferica, che è risultata in quel giorno piuttosto forte; di conseguenza l'immagine della corona non è risultata molto nitida.

La luce diffusa dell'alta atmosfera è stata invece rilevata con sufficiente precisione: infatti gli spostamenti angolari nell'immagine nelle migliori fotografie sono stati dell'ordine di una decina di prini in media.

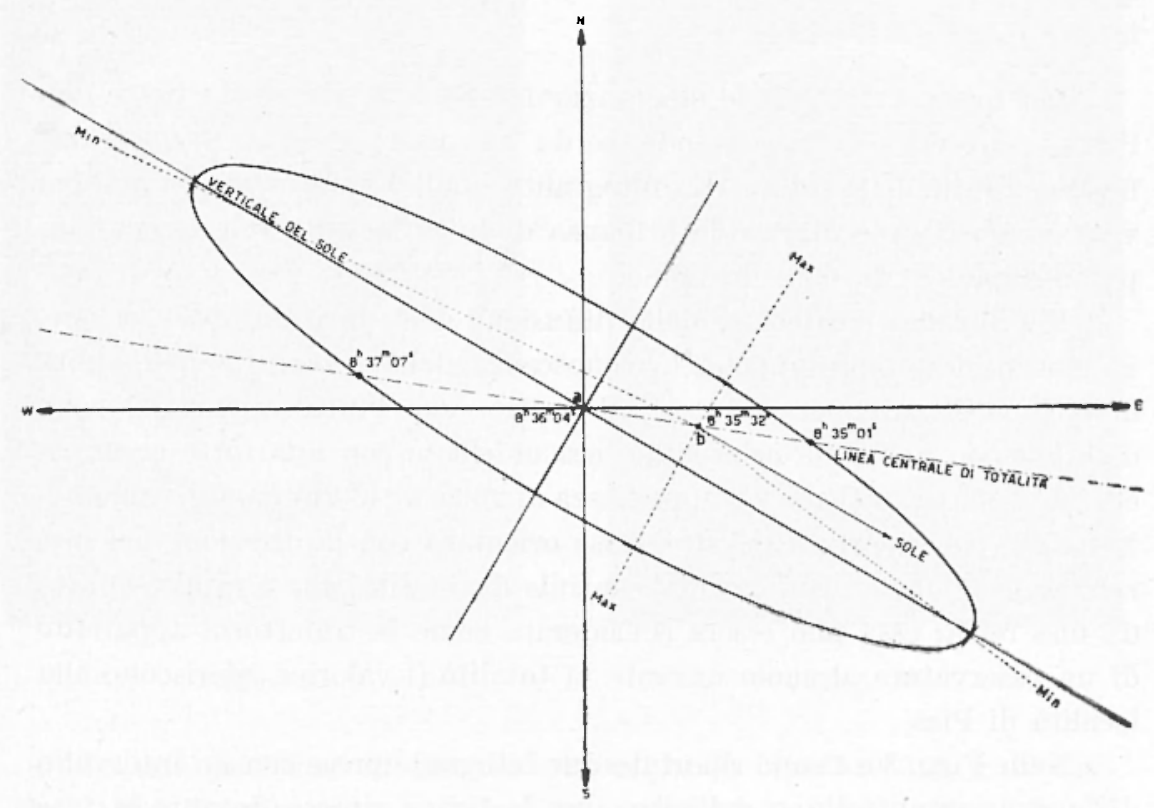

Fig. 2

I risultati che si possono ottenere da questo tipo di rilevamenti fotografici sono tuttora in esame, in quanto sono state scattati un migliaio di fotogranmi durante tutta l'ecelisse. Ma fin d'ora si può senz'altro prevedere un notevole interesse sia dal punto di vista di rilevamento fotografico degli oggetti celesti, in particolar modo della corona solare, sia dal punto di vista delle osservazioni della luce diflusa da parte dell'atmosfera.

Infatti tali rilevamenti, qualowa vengano eseguiti a quote superiori ai $15-20 \mathrm{~km}$ con l'apparato sopra descritto, hanno i seguenti vantaggi:

1) è inutile il puntamento dell'apparecchio fotografico, dato che si tratta di un sistema di ripresa all-sky; 
2) è quasi totalmente annullato l'efietto perturbatore dell'atmosfera sulle immagini degli oggetti celesti;

3) è ridotto notevolmente l'assorbimento delle radiazioni, specie nell'u.r. prossimo;

4) è ridotta la brillanza del cielo diurno, ciò che fa sperare con qualche piccola modifica all'apparato, di poter riprendere la corona solare al difuori dell'eclisse.

Per quanto riguarda le osservazioni della luce diffusa da parte dell'alta atmosfera, un tale metodo, se da una parte presenta le note difficoltà proprie della fotometria fotografica, dall'altra presenta l'indubbio vantaggio di poter rilevare la brillanza di tutta la volta celeste contemporaneamente e in ogni direzione.

Il problema particolare della diffusione della luce durante l'eclisse, da noi studiato, presuppone la conoscenza dellar geometria dell'ombra della luna istante per istante. Nel nostro caso l'intersezione del cono d'ombra con un piano orizzontale era un'ellisse con una forte eccentricità (i semiassi avevano una lunghezza di 200 e di $43 \mathrm{~km}$ rispettivamente. Nella Fig. 2 è riportata questa ellisse orientata con la direzione del meridiano geografico e con la linea centrale di totalità, che è ruppresentata da una retta; essa può essere considerata come la traiettoria apparente di un osservatore al suolo durante la totalità (i valori si riferiscono alla località di Pisib).

Nelle Figg. 3 e 4 sono riportate due fotografie prese con un intervallo di qualche minuto l'una dall'altra, ma la prima ripresa durante la fase parziale e la seconda durante la fase di totalità. Da un semplice esame si può osservare che durante la fase parziale l'atmosferib è nettamente più luminosa nel piano verticale del sole: infatti predomina ancola l'illuminamento diretto della fotosfera solare. Durante la totalità invece si ha solo la luee che proviene dalle zone dell'atmosfera al difuori del cono d'ombra. E nettissima la differenza tra l'illuminamento nel piano verticale del sole (dove la zona luminosa raggiunge l'altezar minima) e l'illuminazione nel piano normale (dove l'altezza è massima). Infatti se si osserva il percorso apparente del pallone nel cono d'ombra, si vede che nella po izione a) (al centro del rono, cioè nella fase massima dell'eclisse) la fascia luminosa dell'atmosfera si trova a ma distanza di circa $43 \mathrm{~km}$ nel piano normale al verticale del sole; cio signifiea che l'altezza della fascia luminosa è di circa $15^{\circ}$ (se si suppone che l'atmosfera sia sensibilmente diffondente fino a quote di 10-15 km); nella direzione 
del sole invece lo stesso angolo vale circa $3^{\circ}$, in quanto la fascia luminosa si trova a una distanza di cirea $200 \mathrm{~km}$. L'altezza di questa fascia luminosa, indipendentemente dal valore numerico si presenta pereió minima nel piano rerticale del sole e massina nel piano and esso normale.

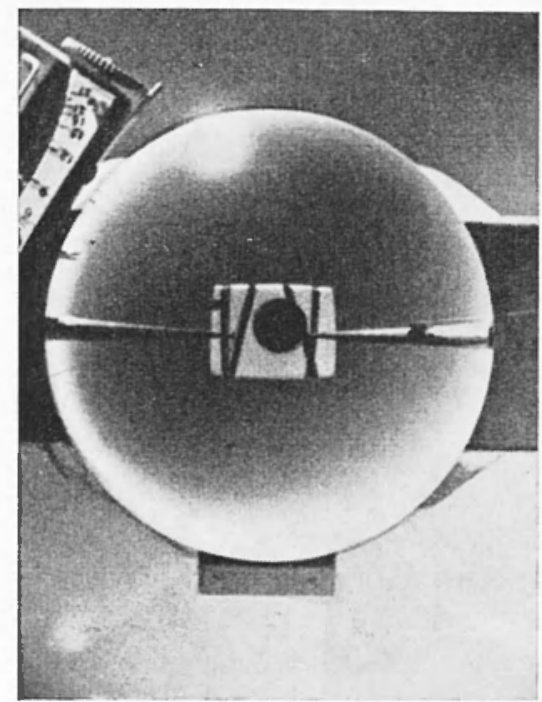

Fig. 3

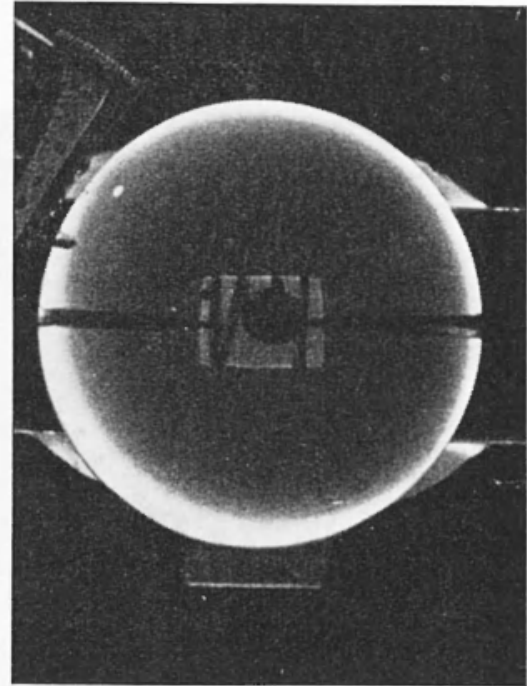

Fig. 4

In una posizione intermedia per es. in $b$ ) la situazione è diversa. Nella Fig. 5 è riportata una fotografia ripresa in questa posizione. Si osserva anzitutto che le altezze della faseia sono essenzialmente diverse, ma è notevole il fatto che la fascia luminosa non ha l'altezza minima nel piano verticale del sole, ma in una direzione sensibilmente diversa. Ciò può essere facilmente interpretato con l'ausilio della Fig. 2 osservando che nella posizione $b$ ) la direzione nella quale la fascia deve avere l'altezzat minima (cioé dove il limite dell'ombra è più distante) non coincide con il piano verticale del sole passante per il pallone.

Le misure fotometriche, che si stamno ora eseguendo, tendono alla valutazione della diflusione della luce da parte dell'alta atmosfera. Laa situazione durante l'eclisse può essere cosi schematizzata: in ogni punto di un fotogramma ripreso durante la totalita si ha un annerimento ehe funzione della brillanza dell'atmosfera nella direzione della volta celeste corrispondente. In ogni direzione questa brillanza è dovuta generalmente a due regioni dell'atmosfera ben distinte: 
1) una regione esterna al cono d'ombra, dove si può considerare prevalente l'illumin umento dovuto alla fotosfera solare;

2) una regione interna al cono d'ombra, dove prevale l'illuminamento dovuto alle regioni dell'atmosfera esterna al cono d'ombra, poichè si può considerare del tutto trascurabile l'illuminamento dovuto alla corona solare.

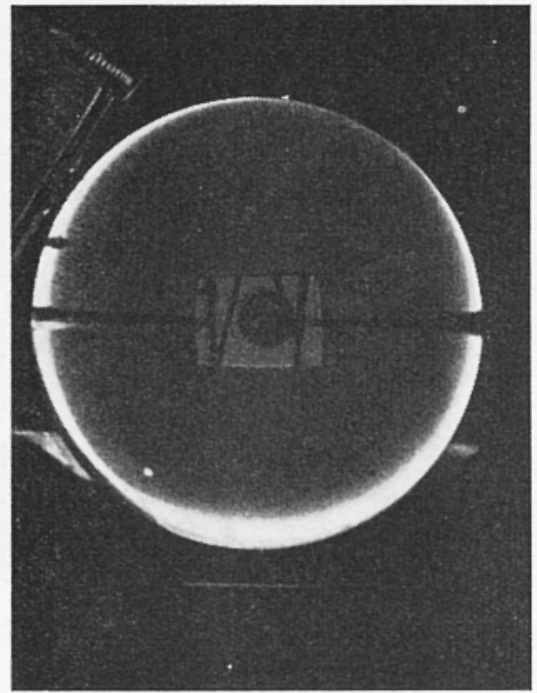

Tig. 5

Le fotografie riprese durante la fase parziale sono state adoperate per la valutazione degli effetti secondari di diffusione dovuti all'atmosfera stessa ed all'alberlo. Un esame preliminare ha già mostrato che, ad ec:cezione di una zona molto bassa vicino all'orizzonte, questi effetti secondari, alle quote da 8.000 ai 10.000 metri circa nelle condizioni dell'attuale eclisse, sono del tutto trascurabili. Pertanto le misure sui fotogrammi scattati durante la totalità permetteranno di nisurare la diffusione secondaria come differenza tra quella osservata e quella primaria dovuta alle regioni 1 ), e che si può calcolare in base alla geometria del cono d'ombra, alla densità dell'atmosfera alle diverse quote ed all'illuminamento dovuto al sole durante la fase parziale. Pеr la realizzazione di questa ricerca, fase iniziale di un programma, che si spera di sviluppare nel prossimo futuro, è gradito ringraziare i Ministeri della Difesa e delle 
Finanze. Essi hamo dato ma sostanziale collaborazione per il laneio a

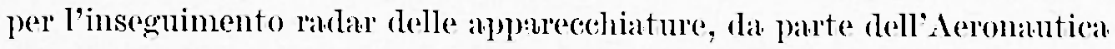
Militare I.T.L.V. D.A.T. a Comando dell'Aeroporto di Pisal e per il recupero delle stesse apparecehiature, eftettuato appena due ore dopo il lancio dallar Sezione Elicotteri dellar Guardial di Finanza di Pisa.

\section{RIASEUTO}

Nel presente lavoro ali A.A. riferiscono su un lancio effettuato durante l'eclisse totale di sole del 15 Frbbraio 1961 mediante palloni. Lo scopo della ricerea $\dot{s}$ qurllo di rilevare la luce diffusa da pate dell atmosfera e di esegnive fotografie della corona esterma del sole ad alta unota.

Gli A.A. deserivono l'apparato adoperato e riportano alcune gotografie ottenute, con una hrere discussione sulla illuminavione della colta celeste durante le fasi dell'ectisss.

\section{$S U H H A R Y$}

On the occasion of the 1961 total solar eclipse a programme of research had been elaborated by the Istituto Nasionale di Geofisica, the Monte Mario Astronomical Observatory and the Italian Ministry of Air meant to measure the light diffused by the atmosphere and to talie photographs of the corona of the sun from high altitudes.

This research aus carried out by sending into the upper atmosphere a photographic camera of the all-sliy type.

In this paper the apparatus are described and some results of this flight are reported with some photographs talien during the eclipse. In order to study the secondary diffusion the illumination of the celestial valt during the eclipse are briefly discussed.

\section{BIBLIOGRAFIA}

(1) R. Cialdesa, Ann. di Geofis., V, 409, (1952).

R. Cialdea, P. Dominici, Ann. di Geofis., V, 581, (1952).

(2) R. Ciat.deA, Ann. di Geofis. IX, 451, (1956). 